\title{
A novel Chk1-binding peptide that enhances genotoxic sensitivity through the cellular redistribution of nuclear Chk1
}

\author{
KWANG SEOK KIM, KYU JIN CHOI and SANGWOO BAE
}

Division of Radiation Effects, Korea Institute of Radiological and Medical Sciences, Seoul 139-706, Republic of Korea

Received March 10, 2016; Accepted September 15, 2016

DOI: $10.3892 /$ ijmm.2016.2762

\begin{abstract}
Since checkpoint kinase 1 (Chk1) is an essential factor for cell viability following DNA damage, the inhibition of Chk1 has been a major focus of pharmaceutical development to enhance the sensitivity of tumor cells to chemo- and radiotherapy that damage DNA. However, due to the off-target effects of conventional Chk1-targeting strategies and the toxicity of Chk1 inhibitors, alternative strategies are required to target Chk1. To facilitate such efforts, in this study, we identified a specific Chk1-binding 12-mer peptide from the screening of a phage display library and characterized the peptide in terms of cellular cytotoxicity, and in terms of its effect on Chk1 activity and sensitivity to genotoxic agents. This peptide, named N-terminal Chk1-binding peptide (Chk1-NP), bound the kinase domain of Chk1. Simulation of the binding revealed that the very $\mathrm{N}$-terminus of the Chk1 kinase domain is the potential peptide binding site. Of note, the polyargininemediated internalization of Chk1-NP redistributed nuclear Chk1 with a prominent decrease in the nucleus in the absence of DNA damage. Treatment with Chk1-NP peptide alone decreased the viability of p53-defective HeLa cells, but not that of p53-functional NCI-H460 cells under normal conditions. The treatment of HeLa or NCI-H460 cells with the peptide significantly enhanced radiation sensitivity following ionizing radiation (IR) with a greater enhancement observed in HeLa cells. Moreover, the IR-induced destabilization of Chk1 was aggravated by treatment with Chk1-NP. Therefore, the decreased nuclear localization and protein levels of Chk1 seem to be responsible for the enhanced cancer cell killing following combined treatment with IR and Chk1-NP. The approach using the specific Chk1-binding peptide may facilitate the mechanistic understanding and potential modulation of Chk1
\end{abstract}

Correspondence to: Dr Sangwoo Bae, Division of Radiation Effects, Korea Institute of Radiological and Medical Sciences, 75 Nowon-Gil, Nowon-Gu, Seoul 139-706, Republic of Korea

E-mail: swbae@kcch.re.kr

Abbreviations: IR, ionizing radiation; DDR, DNA damage response; DDRFs, DNA damage response factors; Chk1, checkpoint kinase 1; Chk1-NP, N-terminal Chk1-binding peptide

Key words: DNA damage response, checkpoint kinase 1, Chk1-binding peptide, genotoxic stress, radiation sensitivity activities and may provide a novel rationale for the development of specific Chk1-targeting agents.

\section{Introduction}

The DNA damage response (DDR), which is caused by genotoxic stresses involves various cellular responses, such as the activation of DNA damage signaling, cell cycle checkpoints, DNA damage repair and cell death (1). The DDR is regulated by concerted activities of the DNA damage responses factors (DDRFs). The DDRFs function as DNA damage sensors, transducers of signaling and effectors for the DDR (2). For example, ataxia telangiectasia mutated (ATM) and ATM-Rad3-related (ATR) initiate DNA damage signaling upon the induction of DNA damage, and trigger a cascade of downstream signaling pathways, which results in the activation of transducing kinases, such as checkpoint kinase 1 (Chk1) and Chk2 (3). The transducing kinases in turn relay the signals to effector molecules, such as CDC25A to regulate the cell cycle checkpoint $(4,5)$.

Chk1 is an essential kinase in the DDR and serves as an important checkpoint regulator in the S- and G2 checkpoints, as well as in the mitotic spindle checkpoint (6). The domain structure of Chk1 shows a highly conserved N-terminal kinase domain and a less conserved C-terminal regulatory domain $(7,8)$. Activity and stability of Chk1 is regulated by its phosphorylation following genotoxic stresses. ATR phosphorylates Chk1 at the highly conserved serine (S)317 and S345 residues (8). The phosphorylation of S345 is commonly used as a biomarker of Chk1 activation. Since the Chk1-mediated checkpoints are crucial for the DDR, protein stability is tightly regulated. The phosphorylation and subsequent activation of Chk1 by ATR-mediated phosphorylation on S317 and S345 lead to conformational changes and the degradation of Chk1 (9). Chk1 is degraded via the ubiquitin-mediated degradation pathway by E3 ubiquitin ligases that employ CUL1 and CUL4A (9-11). As Chk1 activation and modulation of its stability are tightly coupled, the understanding of the processes may be crucial to target Chk1 for cancer therapy. Indeed, considering the essential nature of Chk1 for cell survival, Chk1 has been studied as a promising target for cancer therapy $(12,13)$. Since many cancer cells defective in p53 require the Chk1-induced G2 checkpoint for their survival following genotoxic treatment, the combination of genotoxic stresses and Chk1 inhibitors enhances the efficacy of DNA damaging agents $(14,15)$. A similar effect has also been observed by the combination of Chk1 knockdown 
and ionizing radiation in p53-defective cancer cells (16). As the inhibition of Chk1 enhances tumor cell killing by genotoxic stresses, a number of Chk1 inhibitors, including UCN-01 and AZD7762 have been investigated (12).

In addition to chemical modulators of Chk1, we reasoned that specific Chk1-binding peptides could be delivered into the cells and modulate the activity of Chk1 and Chk1-regulated responses. To achieve this goal, in this study, we screened a phage display library and identified a specific Chk1-binding 12-mer peptide. This peptide specifically binds the N-terminal kinase catalytic domain of Chk1. We report the characterization of the Chk1-binding peptide in terms of intracellular delivery, Chk1-binding and cellular cytotoxicity, as well as in terms of its effect on Chk1 activity and radiosensitivity. Our data may facilitate the development of Chk1-based methods to enhance tumor cell killing by DNA-damaging agents.

\section{Materials and methods}

Expression and purification of the recombinant $N$-terminal fragment of Chkl protein. PCR was used to amplify DNA fragments encoding the N-terminal kinase domain of Chk1 (amino acid residues 2-270) using forward and reverse primers (forward, 5'-CGCGGATCCGCAGTGCCCTTTGTGGAA GACT-3' and reverse, 5'-CGCCAAGCTTACTTGAGGGGTTT GTTGTACCATC-3'). The underlined letters indicate restriction sites for subcloning after PCR steps. The amplification product incorporated a hexahistidine-tag at the beginning of the coding region. An amplified fragment was digested with the BamHI and HindIII restriction enzymes and cloned into a similarly digested pQE30 vector (Qiagen, Venlo, The Netherlands), resulting in a plasmid, which was designated as pQE30Chk1N-His. The His-Chk1 fusion protein was expressed by subjecting the bacteria to $0.1 \mathrm{mM}$ isopropyl-1-thio$\beta$-D-galactopyranoside (IPTG) induction for $6 \mathrm{~h}$. The bacterial extracts containing the expressed Chk1 proteins were subjected to Ni-Affinity chromatography (Qiagen) to the purify N-terminal Chk1 fragment (amino acids 2-270) and subsequently washed with 3 column volumes of $300 \mathrm{mM} \mathrm{NaCl}$ and $20 \mathrm{mM}$ sodium phosphate, $\mathrm{pH}$ 8.0. The protein was eluted with the same buffer containing $300 \mathrm{mM}$ imidazole. The purified Chk1 protein fragment was concentrated, and the protein concentration was determined using the BCA protein assay kit (Thermo Fisher Scientific Inc., Rockford, IL, USA).

Screening of phage display library. The PhD-12 Phage display library kit (\#E8111L; New England Biolabs, Beverly, MD, USA) was used for all panning experiments. A randomized 12-mer peptide was expressed and incorporated into the $\mathrm{N}$-terminus of the pIII coat protein of the M13 bacteriophage separated by a 4 amino acid linker.

A solution of $100 \mu \mathrm{g} / \mathrm{ml}$ of recombinant Chk1 protein in $0.1 \mathrm{M} \mathrm{NaHCO}_{3}(\mathrm{pH}$ 8.6) was added to each well of a 12-well plate and swirled repeatedly until the surface was completely wet followed by incubation overnight at $4^{\circ} \mathrm{C}$ with gentle agitation in a humidified container. Phages $\left(2 \times 10^{11} \mathrm{pfu}\right)$ were added to the recombinant Chk1-coated plate after blocking with $1 \mathrm{ml}$ $5 \%$ skimmed milk for $1 \mathrm{~h}$ at $25^{\circ} \mathrm{C}$. The mixture was incubated for $2 \mathrm{~h}$ at $25^{\circ} \mathrm{C}$ with gentle shaking. Unbound phages were removed by approximately 6 washes with TBST buffer $(50 \mathrm{mM}$ Tris- $\mathrm{HCl}$
$\mathrm{pH} 7.5,150 \mathrm{mM} \mathrm{NaCl}$ with $0.1 \%$ Tween-20). The bound phage particles were eluted with $0.2 \mathrm{M}$ glycine- $\mathrm{HCl}$ ( $\mathrm{pH} 2.2$ ), neutralized with $1 \mathrm{M}$ Tris- $\mathrm{HCl}$ ( $\mathrm{pH}$ 9.1), and used for the titer assay and amplification in E.coli ER2738. The phage titer was evaluated by the blue plaque-forming assay on an agar plate containing IPTG and X-gal. To amplify the selected phage clones, the phages were mixed with $20 \mathrm{ml}$ E. coli $\mathrm{ER} 2738$ culture and incubated at $37^{\circ} \mathrm{C}$ with vigorous shaking for $4.5 \mathrm{~h}$. The phage particles were then harvested, resuspended in $50 \mu 1$ TBS $(50 \mathrm{mM}$ Tris- $\mathrm{HCl}$ $\mathrm{pH} 7.5,150 \mathrm{mM} \mathrm{NaCl}$ ) with $0.02 \%$ sodium azide and titred as per the manufacturer's instructions. Round 2 and 3 of the selections with $2 \times 10^{10}$ and $2 \times 10^{9}$ phages, respectively, which were harvested from the first selection, were performed with recombinant protein-coated plates. The above-mentioned procedures were repeated for the specific Chk1-binding peptides. Following 3 rounds of positive panning, 50 single plaques were selected for DNA sequencing.

Peptide pull-down assay. Biotin-labeled peptides were purchased from Peptron (Daejeon, Korea). The R9 and N-terminal Chk1-binding peptide (Chk1-NP) peptide have the following amino acid sequences, R9, Biotin-Arg-Arg-ArgArg-Arg-Arg-Arg-Arg-Arg; and Chk1-NP, Biotin-Arg-ArgArg-Arg-Arg-Arg-Arg-Arg-Arg-Ala-Pro-Asn-Lys-Thr-Leu-SerVal-Asn-Lys-Met-Val. The peptides were dissolved in distilled water. HeLa (ATCC ${ }^{\circledR}$ CCL-2TM; ATCC, Manassas, VA, USA) were treated with $5 \mu \mathrm{M}$ of each peptide followed by incubation at $37^{\circ} \mathrm{C}$ for $6 \mathrm{~h}$. To prepare cell extracts, the cells were lysed in an IP buffer (1\% Triton X-100, $50 \mathrm{mM}$ Tris-Cl, pH 7.4, $300 \mathrm{mM}$ $\mathrm{NaCl}, 5 \mathrm{mM}$ EDTA) containing protease inhibitors. A highspeed centrifugation step removed the cell debris from the cell lysates. An equal amount $(1 \mathrm{mg})$ of cell extracts was used in the pull-down assay. Pull-down assay for the biotinylated peptide and its interacting Chk1 was carried out by following manufacturer's instructions (Pierce ${ }^{\mathrm{TM}}$ Biotinylated protein interaction pull-down kit \#21115; Thermo Scientific, Inc.). Specific peptide-bound Chk1 was detected by western blot analysis using anti-Chk1 antibody (mouse monoclonal antibody against human Chk1, sc-8408; Santa Cruz Biotechnology, Inc., Dallas, TX, USA). Western blot analysis was performed as previously described (17).

Western blot analysis. Specific peptide-bound Chk1 was detected by western blot analysis using anti-Chk1 antibody (mouse monoclonal antibody against human Chk1, sc-8408; Santa Cruz Biotechnology, Inc.). Western blot analysis was performed as previously described (17). Briefly, protein extracts (35 mg) were separated on $10 \%$ SDS-polyacrylamide gels and then transferred onto nitrocellulose membranes. The membranes were incubated with 5\% skim milk solution in Tris-Tween Buffered Saline (TTBS) solution overnight at $4^{\circ} \mathrm{C}$ with the indicated antibodies. After washing 3 times in TTBS, horseradish peroxidase-conjugated secondary antibodies were applied. The proteins were visualized using enhanced chemiluminescence (GE Healthcare Life Sciences, Pittsburgh, PA, USA). $\beta$-actin is a control for protein loading and detected in western blot analysis using a specific antibody (sc-81178; Santa Cruz Biotechnology, Inc.).

Fluorescence microscopy. The HeLa cells were cultured on sterile coverslips in a 12 -well plate. The cells were incubated 


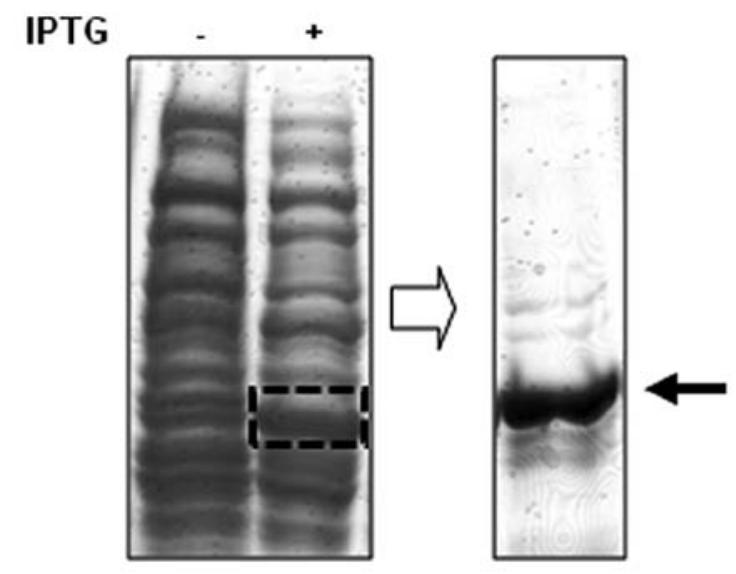

Figure 1. Isolation of the recombinant N-terminal fragment of checkpoint kinase 1 (Chk1) (aa 2-270). Hexahistidine-tagged Chk1 fragment (amino acid 2-270) was isolated from bacterial extracts subjected to IPTG induction. The arrow indicates the isolated Chk1 N-terminal fragment.

with the peptides $\left(5 \mu \mathrm{M}\right.$ of $\mathrm{R} 9$ or Chk1-NP) for $2 \mathrm{~h}$ at $37^{\circ} \mathrm{C}$ in $5 \% \mathrm{CO}_{2}$ humidified air and washed 3 times with phosphatebuffered saline (PBS; Thermo Fisher Scientific Inc.). The cells were then fixed with methanol, stained with anti-Chk1 antibody (mouse monoclonal antibody against human Chk1, sc-8408; Santa Cruz Biotechnology, Inc.) and Alexa Fluor 594 goat anti-mouse secondary antibody (A-11005; Invitrogen ${ }^{\mathrm{TM}}$, Grand Island, NY, USA), washed with PBS, and re-stained with FITC-conjugated streptavidin (SA100-02) (both from Invitrogen) before staining with 4',6-diamidino-2-phenylindole (DAPI). The nuclei were fluorescently labeled with DAPI (D9542; Sigma-Aldrich, Inc., St. Louis, MO, USA). The cells were then imaged using a Leica DMIRB microscope (Leica Microsystems Inc., Wetzlar, Germany) with a X20 objective. The fluorescent images were merged. HJURP was also detected by fluorescence microscopy as a nuclear resident protein and compared with localization of Chk1.

Cell viability assay. The HeLa or NCI-H460 (ATCC) were treated with the R9 or Chk1-NP peptide, or no peptide as a control; 0, 5,10 or $20 \mu \mathrm{M}$ of the peptides were used for cell treatment. To measure cell survival, the peptide-treated cells were incubated up to $48 \mathrm{~h}$. Cell viability was measured by MTT assay as previously described (17). For combination treatment of the cells with peptide and genotoxic stresses, the HeLa or NCI-H460 cells were used. The cells were treated with $5 \mu \mathrm{M}$ of the R9 or Chk1-NP peptide for $1 \mathrm{~h}$ pior to treatment with genotoxic agents, such as $500 \mathrm{nM}$ camptothecin (CPT; \#C9911) $5 \mathrm{mM}$ hydroxyurea (HU; \#H8627) (both from Sigma-Aldrich) or $10 \mathrm{~Gy}$ ionizing radiation (IR). Cell survival was measured by MTT assay after $24 \mathrm{~h}$. To measure and compare effects of the peptides on the radiation sensitivity of the different cell lines, the HeLa or NCI-H460 cells were treated with $5 \mu \mathrm{M}$ of the R9 or Chk1-NP peptide for $1 \mathrm{~h}$ prior to treatment with 5 Gy IR. MTT assay was used to measure cell viability after $24 \mathrm{~h}$. Cell cultures were irradiated using a 137Cs gamma-ray source (Atomic Energy of Canada, Ltd., Chalk River, ON, Canada) at a dose rate of $3.81 \mathrm{~Gy} / \mathrm{min}$.

$3 D$ modeling to predict the Chkl-NP peptide-binding surface of Chk1. The PEP-Site Finder program was used to identify candidate sites of peptide interactions on the Chk1 protein surface, as previously described (18). To use the program, data of the 3D structure of Chk1 (PDB ID: 2E9N) and peptide sequence information (APNKTLSVNKMW) were used as inputs. The optimal peptide-binding Chk1 surface was generated. Information about the $2 \mathrm{D}$ and $3 \mathrm{D}$ structure of the Chk1 kinase domain was obtained from the RCSB Protein Data Bank (www.rcsb.org).

Statistical analysis. All analyses were carried out using the two-tailed Student's t-test. The means of 3 independent experiments were graphed and data are presented as the means \pm standard deviation. A value of $\mathrm{P}<0.05$ was considered to indicate a statistically significant difference.

\section{Results}

In order to screen specific Chk1-binding peptides, we first isolated the recombinant Chk1 protein fragment to use as a target for potential binding peptides (Fig. 1). The recombinant fragment of Chk1 protein is the N-terminal portion of Chk1 protein which is composed of amino acid residues from 2 to 270 of Chk1. A 6-mer histidine-tagged Chk1 fragment was isolated from the bacterial extracts subjected to IPTG induction (Fig. 1).

The N-terminal Chk1 fragment encompasses the Chk1 kinase domain as shown in Fig. 2A. Moreover, the secondary structure of the N-terminalChk1 fragment is mainly composed of $\alpha$-helices and $\beta$-sheets $(19,20)$. A Chk1 fragment-bound 12-mer peptide was screened from the phage display library, which has an amino acid sequence of APNKTLSVNKMV (Fig. 2B).

Simulation of the 12-mer peptide-binding to the N-terminal Chk1 fragment revealed potential sites of Chk1 for the peptide binding (Fig. 2B). Models of the peptide Chk1 binding predict that the very $\mathrm{N}$-terminus of $\mathrm{Chk} 1$, which is composed of mostly a $\beta$-sheet secondary structure is the peptide-binding site of Chk1. In order to test the activity of the peptide in the cell, we used a 9-mer poly-arginine sequence for an internalization domain. A poly-arginine peptide (R9 peptide) (Fig. 2C) has been shown to efficiently transport peptides and proteins into mammalian cells (21). We used two different peptides, such as an R9 internalization domain alone peptide as a control and a specific Chk1-binding peptide, named Chk1-NP, which is composed of R9 plus the identified Chk1-binding 12-mer peptide sequence (Fig. 2C). These two peptides were tagged with a biotin at their $\mathrm{N}$ termini for easy detection in in vitro experiments. In order to confirm the Chk1-binding activity of the identified 12-mer peptide, we carried out a pull-down experiment with cell extracts and the two peptides. Immunoblot detection of the precipitated Chk1 demonstrated that only the Chk1-NP peptide was able to bind Chk1, but not the R9 control peptide (Fig. 2D).

To explore the activities of the peptides in the cells, we examined the delivery of the fusion peptides into the cell. Treatment of the HeLa cells with R9 or Chk1-NP at a concentration of $5 \mu \mathrm{M}$ showed a significant cellular uptake of the peptides (Fig. 3A). Detection of the cellular localization of the biotin-labeled peptides by FITC-conjugated streptavidin revealed cytoplasmic and nuclear localization of the peptides, indicating that the peptide could be efficiently delivered into 


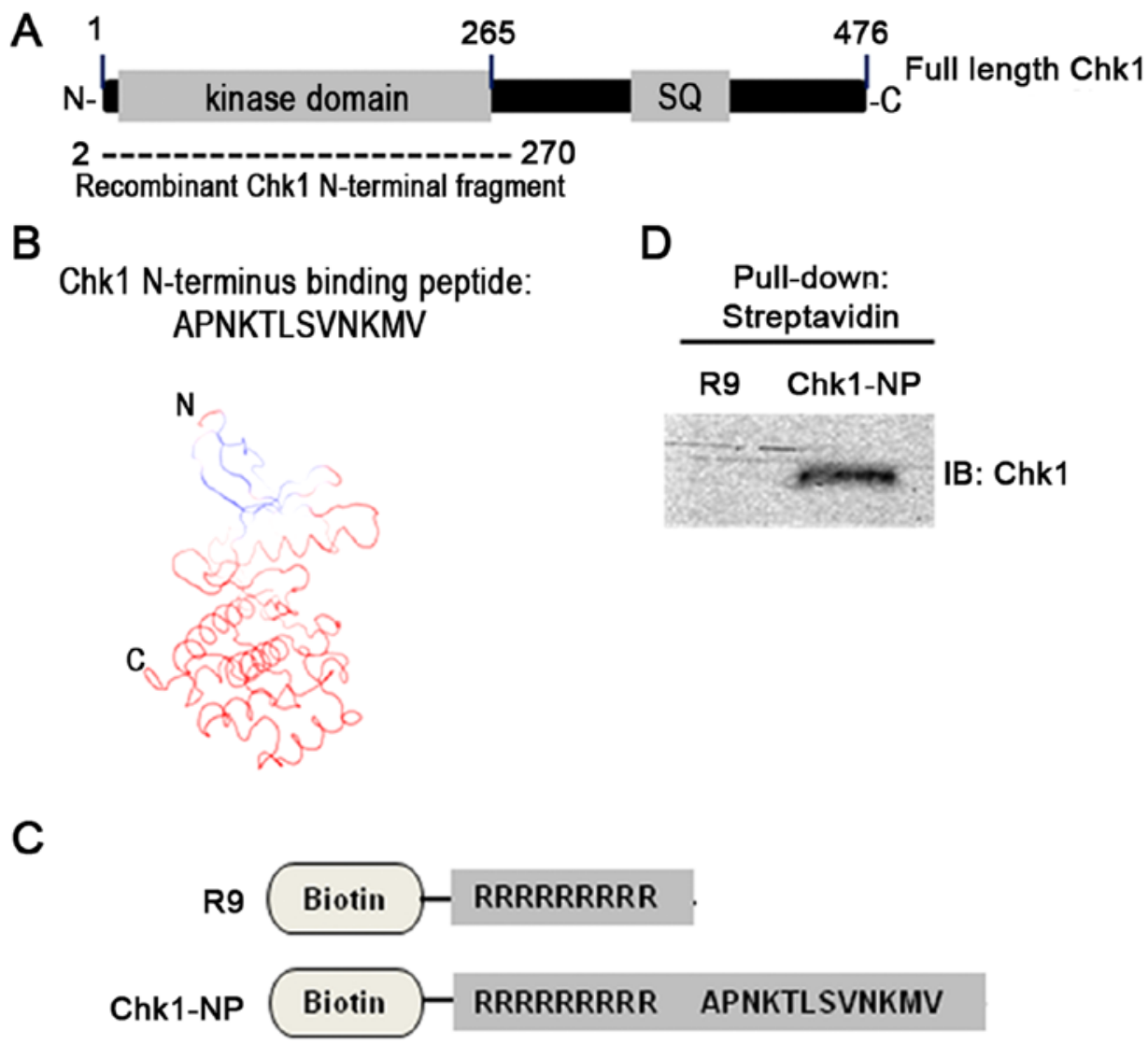

Figure 2. Identification of a peptide that can specifically bind to the N-terminus of checkpoint kinase 1 (Chk1). (A) Domain structure of Chk1 (human full length, amino acid 1-476). The kinase domain (amino acid 8-265) and SQ motif are indicated. The SQ motif is a cluster of serine and glutamine residues that is phosphorylated following DNA damage. The SQ motif encompasses serine 317 and 345 residues that are phosphorylated by ATR. Dotted line indicates the N-terminal fragment of Chk1 (amino acid 2-270) which is expressed as a recombinant protein and used as a bait in the screening of a phage display library. (B) Amino acid sequence of the screened 12-mer Chk1 N-terminal binding peptide (APNKTLSVNKMV) and potential sites of the peptide interactions on the Chk1 N-terminal fragment (PDB ID: 2E9N). Candidate sites of peptide interactions on the Chk1 fragment are presented in blue, which are the very N-terminal $\beta$-sheets in the Chk1 fragment. PEP-Site Finder program was used to find potential sites of peptide interactions on Chk1. (C) Biotin-labeled peptides for experiments. R9 has biotin-lebeled 9-mer polyarginine peptide and serves as a control. N-terminal Chk1-binding peptide (Chk1-NP) is a fusion of biotin-labeled 9-mer polyarginine and 12-mer peptide that can bind the Chk1 N-terminal fragment. 9-mer polyarginine is used for internalization of the peptides. (D) Interaction of Chk1-NP with Chk1. Pull-down assay of the biotinylated peptides with streptavidin beads and subsequent western blot analysis revealed a specific interaction between Chk1-NP and Chk1.

the cellular compartments. Of the cellular compartments, the nuclear staining was more intense than that of the cytoplasm, which may reflect more densely populated peptides in the nucleus. The localization of Chk1 was also examined in the presence or absence of the peptides (Fig. 3B). As shown in Fig. 3A, the peptides, R9 and Chk1-NP, were shown mainly in the nucleus, whereas the no-peptide control $(\mathrm{C})$ cells showed no fluorescence, as expected (Fig. 3B). Chk1 was visible both in the cytoplasm and nucleus, which is consistent with a previous study (22). The nuclear staining of Chk1 is dense compared to the diffuse cytoplasmic staining. The no-peptide control (C) or R9-treated control (R9) exhibited clear nuclear staining of Chk1. However, Chk1 staining in the Chk1-NP treated cells showed a sparse nuclear staining compared to the cytoplasmic staining (Fig. 3B). The sparse nuclear staining of Chk1 may imply that Chk1-NP redistributes nuclear Chk1 to other cellular compartments.

To examine the Chk1 localization further, we compared the localization of Chk1 and HJURP in the R9 or Chk1-NP peptide-treated cells. HJURP, a deposition factor of CENP-A at centromeres, is a nuclear protein (23). The cellular localization of HJURP was unaffected by the R9 and the Chk1-NP peptide, as it showed consistent nuclear staining (Fig. 3C). By contrast, as shown in Fig. 3B, the nuclear staining of Chk1 in the Chk1-NP peptide-treated cells was prominently weak when compared to that of the R9 control peptide-treated cells (Fig. 3C). This finding indicates that Chk1-NP redistributes nuclear Chk1 in a Chk1-specific manner.

Since Chk1 is a critical component in cellular DNA damage responses, we wished to determine whether the Chk1-binding peptide has an effect on the function of Chk1 following treatment with genotoxic stresses. We first evaluated the cytotoxicity of the R9 and Chk1-NP peptides in NCI-H460 or in HeLa cells (Fig. 4A and B, respectively). The two cell lines have a different genetic background, in that NCI-H460 cells are functional in p53, whereas HeLa cells are not.

NCI-H460 cell survival was unaffected by treatment with either peptide at a concentration of up to $20 \mu \mathrm{M}$ (Fig. 4A). By contrast, the survival of the HeLa cells exhibited a striking difference between the peptides used (Fig. 4B). Whereas the R9 control peptide had no effect on HeLa cell survival, the Chk1-binding Chk1-NP peptide decreased cell survival 
A

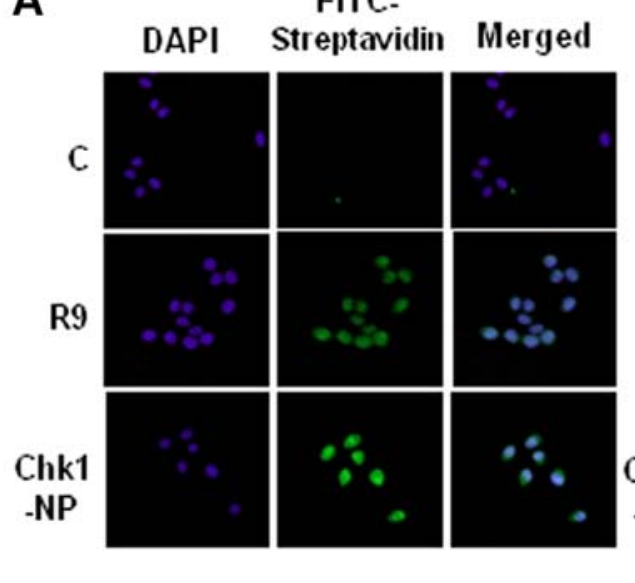

B

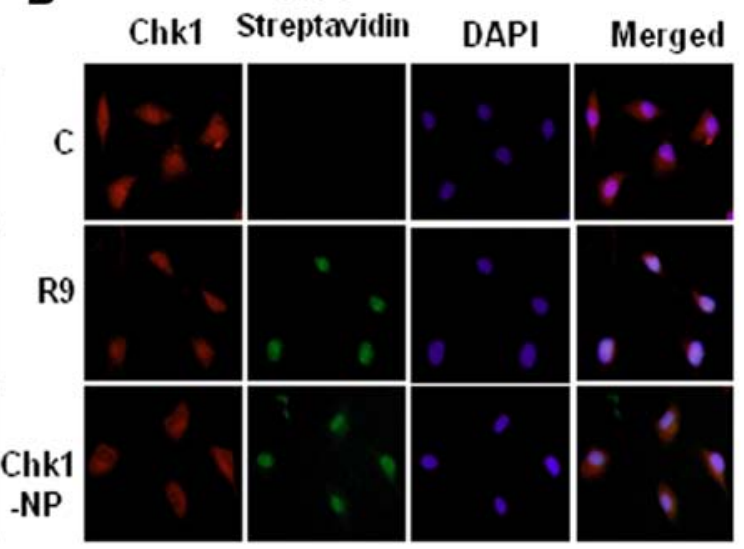

C
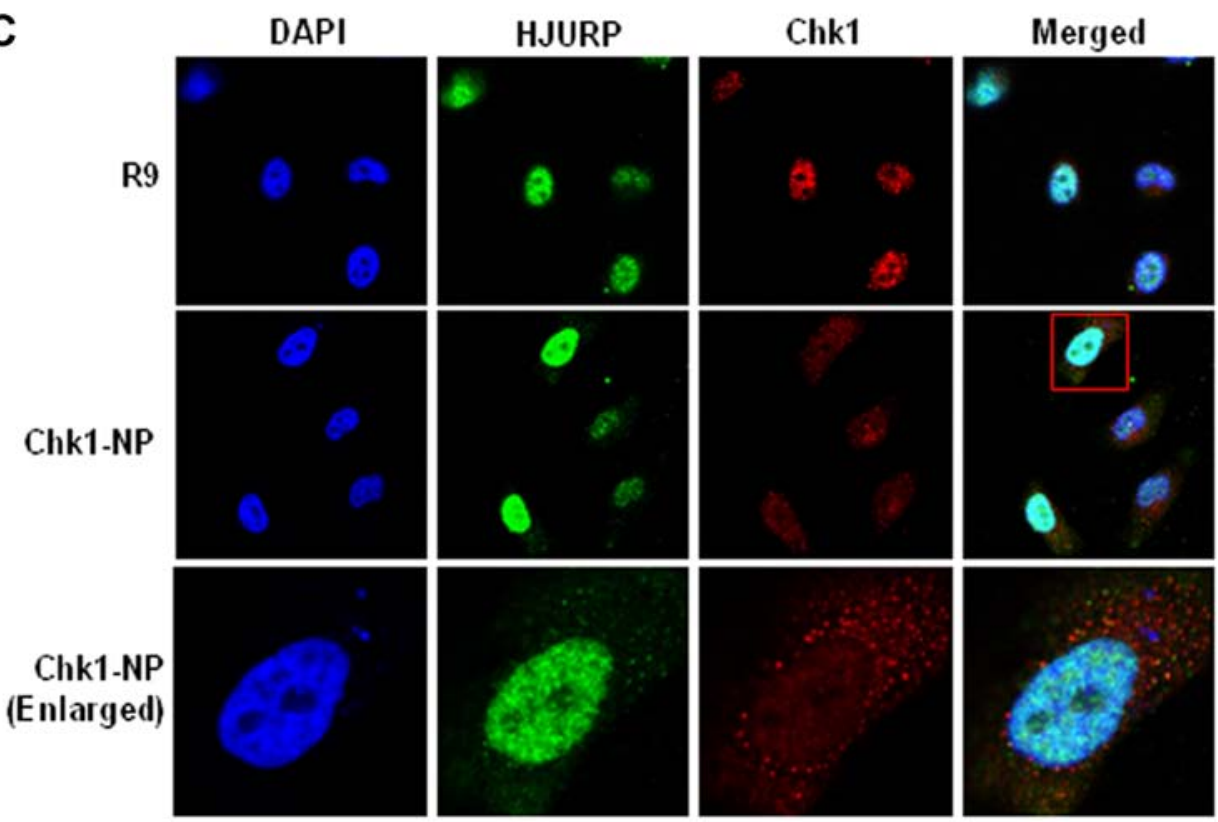

Figure 3. Peptide internalization and its effect on the cellular distribution of checkpoint kinase 1 (Chk1). (A) HeLa cells were treated with $5 \mu \mathrm{M}$ R9 or N-terminal Chk1-binding peptide (Chk1-NP) for $2 \mathrm{~h}$ and analyzed by immunofluorescence microscopy. FITC-conjugated streptavidin was used to detect biotinylated peptides. C is a no-peptide treatment control. Nuclear staining by DAPI is also shown. (B) Effect of the peptides on Chk1 localization. HeLa cells were treated as described in (A). A prominent decrease in the nuclear pool of Chk1 is observed in Chk1-NP treated cells. (C) Decreased nuclear localization of Chk1 by Chk1-NP treatment. Nuclear localization of endogenous Chk1 is significantly decreased only in Chk1-NP treated cells. HJURP, a nuclear resident protein, is unaffected by the peptides. The image in the inset was enlarged in the panel below.

by approximately $30 \%$ at a concentration of $20 \mu \mathrm{M}$ of the peptide (Fig. 4B). This finding may imply that the essential function of Chk1 for HeLa cell survival was affected by the Chk1-binding peptide, Chk1-NP. Moreover, the results suggest that the effect of the Chk1-binding peptide on cell survival may be cell type-specific or more specifically, genotype-specific.

HeLa cells are non-functional in p53, and have been reported to be dependent on Chk1 function for cell survival following exposure to genotoxic stress. Therefore without Chk1 function, HeLa cells are vulnerable to genotoxic stresses and undergo increased cell death by caspase-2 activation (24). In this study, we examined whether Chk1-NP has an effect on HeLa cell survival following treatment with various genotoxic stresses (Fig. 4C). Treatment with CPT, HU, or with IR decreased the survival of the HeLa cells. Whereas decrease in HeLa cell survival was observed in the cells treated with either the R9 or Chk1-NP peptides following exposure to genotoxic stresses, the decrease was more prominent in the cells treated with Chk1-NP (Fig. 4C). The survival of the HeLa cells treated with Chk1-NP was about half that of the R9-treated cells following treatment with IR (Fig. 4C). These findings suggest that Chk1-NP interferes with the functions of Chk1 and significantly increases the death of vulnerable cells, such as HeLa cells following DNA damage.

We further carried out a comparison between the HeLa and NCI-H460 cells as regards radiation sensitivity following treatment with the peptides (Fig. 5A). Both the HeLa and NCI-H460 cells showed a significantly increased radiation sensitivity following treatment with the Chk1-NP peptide. The survival of the Chk1-NP-treated HeLa and NCI-H460 cells following exposure to IR was approximately 35 and $50 \%$, respectively when compared to that of no peptide control (NC) or the R9 

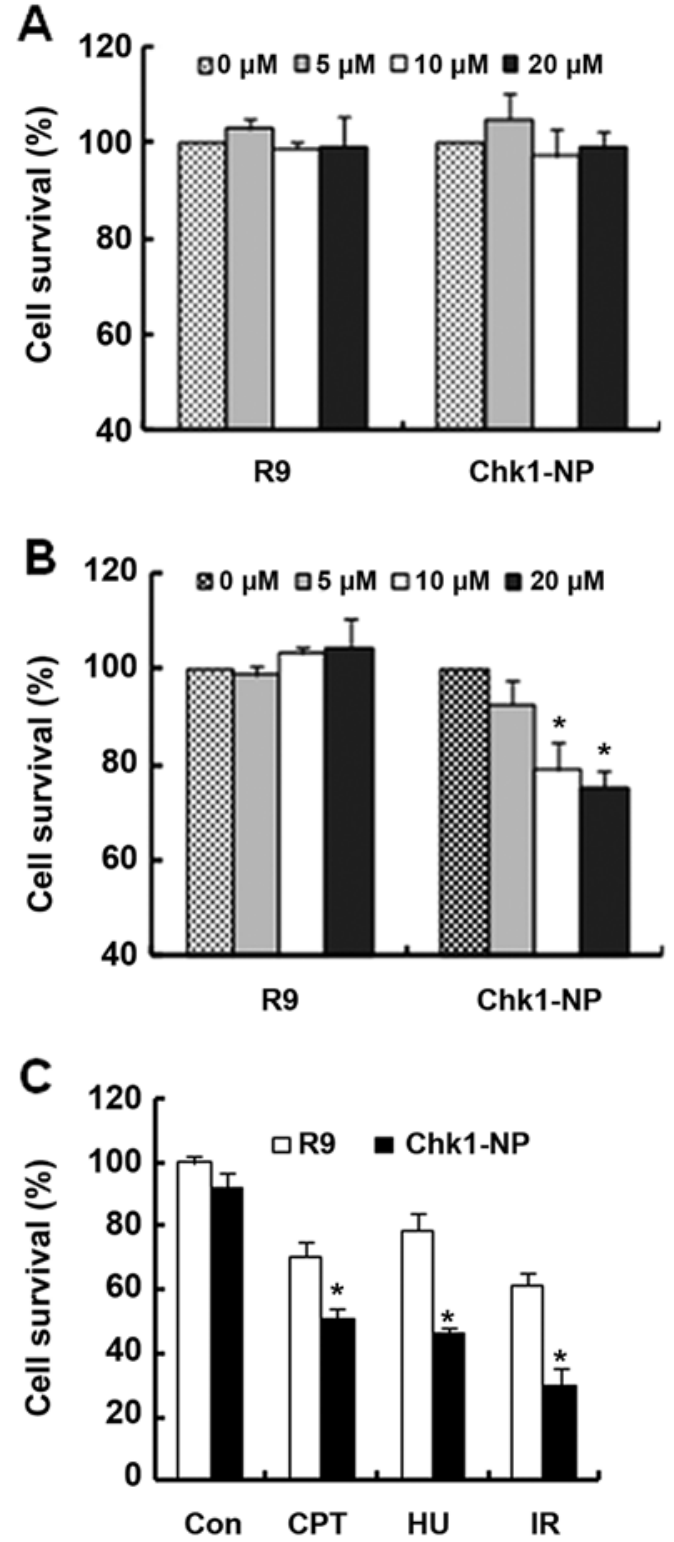

Figure 4. Effect of the R9 or N-terminal Chk1-binding peptide (Chk1-NP) on cell viability. (A) Viability of NCI-H460 cells was measured by MTT assay folllowing treatment with $0,5,10$ or $20 \mu \mathrm{M}$ of R9 or Chk1-NP. Peptide-treated cells were incubated up to $48 \mathrm{~h}$. (B) Viability of HeLa cells was measured by MTT assay following the same treatment as (A). (C) Effect of R9 or Chk1-NP on viability of HeLa cells following genotoxic treatments. Cells were treated with $5 \mu \mathrm{M}$ of R9 or Chk1-NP for $1 \mathrm{~h}$ prior to treatment with genotoxic agents such as $500 \mathrm{nM}$ camptothecin (CPT), $5 \mathrm{mM}$ hydroxyurea (HU) or $10 \mathrm{~Gy}$ ionizing radiation (IR). Cell survival was measured by MTT assay after $24 \mathrm{~h}$. Con stands for control. Error bars represent the means \pm tandard deviation. The means of 3 independent experiments were graphed. ${ }^{*} \mathrm{P}<0.05$ compared to R9-treated group.

control-treated cells. Thus, these findings demonstrate that Chk1-NP is able to enhance the radiation sensitivity of the cells with a greater enhancement observed in p53-defective cancer cells, such as HeLa cells.

Examination of Chk1 phosphorylation in the Chk1-NPtreated HeLa cells following expsoure to IR showed increased the phosphorylation of Chk1 S345 residue (pS345 Chk1) when compared to that of the $\mathrm{NC}$ or the R9 control-treated cells (Fig. 5B). The results are reminiscent of the findings that the Chk1 inhibitor, AZD7762, enhanced chemosensitization
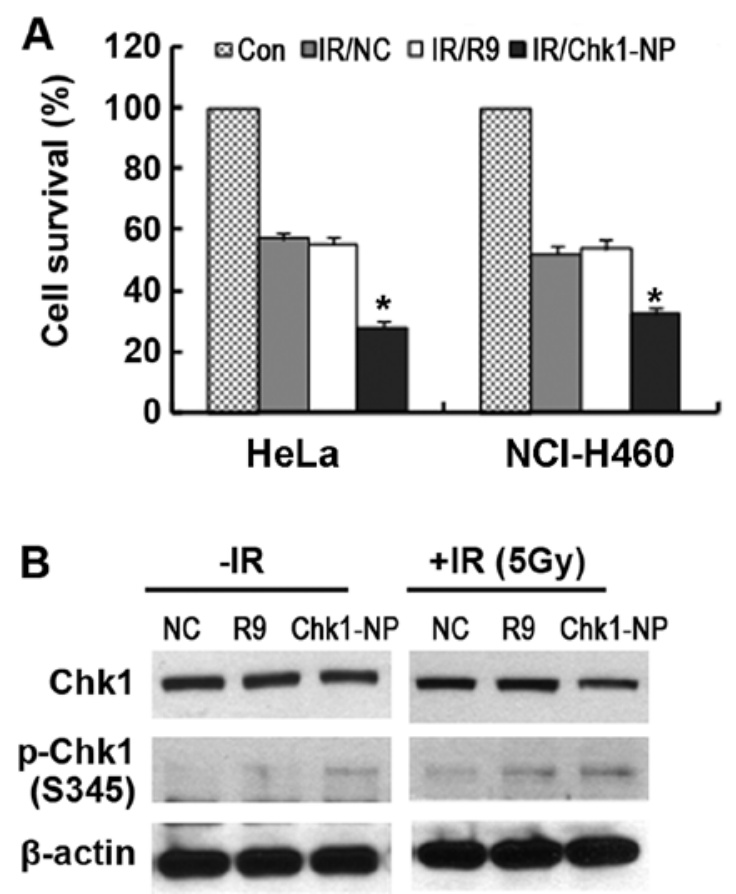

Figure 5. Effect of R9 or N-terminal Chk1-binding peptide (Chk1-NP) on the radiosensitivity of HeLa and NCI-H460 cells. (A) HeLa or NCI-H460 cells were treated with $5 \mu \mathrm{M}$ of $\mathrm{R} 9$ or Chk1-NP peptide for $1 \mathrm{~h}$ prior to treatment with $5 \mathrm{~Gy}$ IR. MTT assay was used to measure cell viability after $24 \mathrm{~h}$. Con stands for control. The means of 3 independent experiments were graphed. (B) Effect of R9 or Chk1-NP on Chk1 activation following exposure to IR. HeLa cells were treated as described in (A). Western blot analysis of Chk1 and phosphorylated (p-)Chk1 (S345) was carried out. $\beta$-actin was used as a loading control. ${ }^{*} \mathrm{P}<0.05$ compared to only IR or IR/R9-treated group.

with a concomitant induction of $\mathrm{pS} 345 \mathrm{Chk} 1$ (25). We also noted that the total levels of Chk1 in the Chk1-NP-treated HeLa cells were less than those in the $\mathrm{NC}$ or the $\mathrm{R} 9$ control-treated cells following exposure to IR (Fig. 5B). Since Chk1 is degraded in a Chk1 phosphorylation-dependent manner following exposure to IR, these findings suggest that the Chk1-NP peptide is able to facilitate the activation-induced degradation of Chk1 following exposure to IR. Furthermore, our results suggest the potential use of Chk1-NP in the artificial modulation of Chk1 stability.

\section{Discussion}

Since Chk1 is essential for cell survival following DNA damage, a mechanistic understanding and potential modulation of Chk1 is of importance to cancer therapy. To facilitate such efforts, in this study, we screened and identified a specific Chk1-binding peptide. This peptide, named Chk1-NP, bound the N-terminal kinase domain of Chk1. Simulation of the peptide binding revealed that the very $\mathrm{N}$-terminus of the Chk1 kinase domain was the potential peptide binding site. The effect of the Chk1-NP peptide on endogenous Chk1 was examined following internalization of the peptide. Cell viability was significantly decreased by combined treatment with the peptide and genotoxic agents. Our findings suggest that the decreased nuclear localization of endogenous Chk1 following the internalization of the Chk1-NP peptide is a likely cause of the decreased cell survival following genotoxic treatments. 

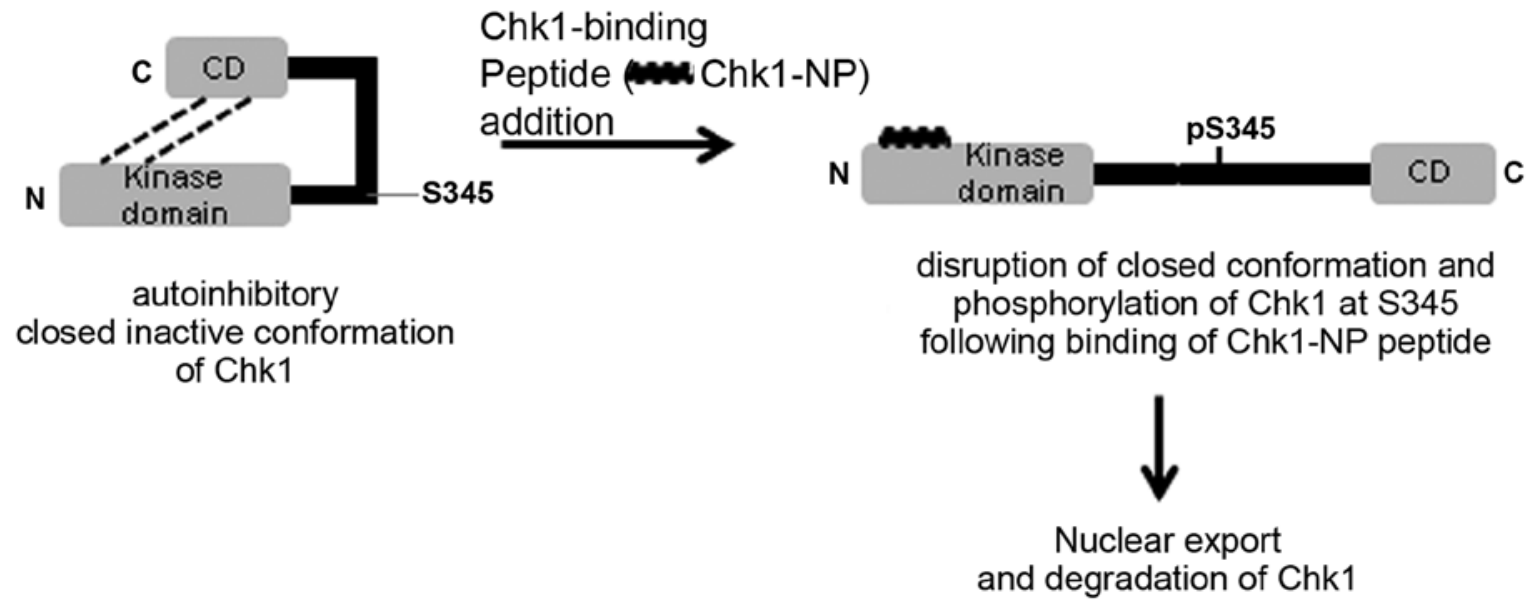

Figure 6. Proposed model for conformational regulation of checkpoint kinase 1 (Chk1) by N-terminal Chk1-binding peptide (Chk1-NP). Autoinhibitory closed conformation of Chk1 is maintained by the intramolecular interaction between the N-terminal kinase domain and the $\mathrm{C}$-terminal domain (CD). Binding of Chk1-NP to the very N-terminal part of the Chk1 kinase domain disrupts the closed conformation and releases the C-terminal domain. Serine (S)345 residue is phosphorylated and the pChk1 is exported to the cytoplasm. Open conformation of Chk1 upon Chk1-NP binding may facilitate the phosphorylation of Chk1 at S345, which leads to the activation of Chk1. pChk1 may follow the following fates: transduction of Chk1 signaling to downstream targets, export to the cytoplasm and its degradation. Therefore Chk1-NP may enhance radiation sensitivity by changing conformation, localization, activity and the protein stability of Chk1.

In the absence of genotoxic stresses, Chk1 seems to undergo an intramolecular interaction between the N-terminus and the C-terminus (Fig. 6) (26). By this interaction, Chk1 blocks the active site of the kinase domain of Chk1, which results in autoinhibition $(11,26)$. Upon DNA damage, Chk1 is phosphorylated by ATR on chromatin. Chk1 phosphorylation seems to release the protein from the autoinhibitory conformation and leads to activation of the protein. Moreover, upon Chk1 phosphorylation, the protein is released from the chromatin and moves into the soluble nucleoplasm and later into the cytoplasm $(9,27,28)$. Considering these facts, we hypothesized the mechanisms through which Chk1-NP may affect multiple aspects of Chk1 activities. These may involve peptide bindinginduced conformational changes and the cellular redistribution of Chk1 (Fig. 6). The Chk1-binding peptide, Chk1-NP may affect the conformation of endogenous Chk1, and this in turn may lead to the decreased nuclear localization of Chk1. Since our simulation of the peptide binding to Chk1 revealed that the very $\mathrm{N}$-terminus of the Chk1 kinase domain was the potential peptide binding site (Fig. 2B), the closed inactive conformation of Chk1 may be released by the disruption of the intramolecular interaction upon binding of Chk1-NP peptide to the N-terminus of Chk1 (Fig. 6). This conformational change may expose the C-terminus of Chk1 and lower the threshold for Chk1 activation to Chk1-activating kinases upon DNA damage. Of note, treatment of the cells with the Chk1-binding peptide alone without DNA damage slightly increased Chk1 phosphorylation at the Ser345 residue (Fig. 5B). This finding is reminiscent of that of a previous study, where the disruption of the closed conformation of Chk1 resulted in Chk1 activation even in the absence of DNA damage (29). In that study, the authors showed that the N-terminal kinase domain of Chk1 prevents Chk1 phosphorylation at the C-terminus by ATR in the absence of DNA damage. Disruption of the closed inactive conformation of Chk1 by mutation of C-terminal conserved residues and loss of the inhibitory effect by the N-terminus result in phosphoryla- tion of Chk1 by ATR even in the absence of DNA damage (29). We speculate that the Chk1-binding peptide causes similar effect as the Chk1 mutation in disrupting closed conformation of Chk1. A specific binding of Chk1-NP to the Chk1 $\mathrm{N}$-terminus may disrupt the closed conformation of Chk1 and release the $\mathrm{C}$-terminus from the inhibitory $\mathrm{N}$-terminus which may lead to Chk1 activation by phosphorylation. Irradiation of the Chk1-NP peptide-treated cells increased further Chk1 phosphorylation at the S345 residue, which is an indication of Chk1 activation (Fig. 5B). Open conformation of Chk1 upon Chk1-NP binding may have facilitated the phosphorylation of Chk1 following IR (Fig. 6).

As conformational changes of Chk1 are closely tied to its spatial redistribution $(9,27,28,30)$ we may expect changes in Chk1 localization following Chk1-NP peptide binding. Chk1-NP significantly decreased the nuclear localization of endogenous Chk1 (Fig. 3C). Chk1 phosphorylation triggers a release of Chk1 from chromatin upon DNA damage into the nucleoplasm and the cytoplasm $(9,27,28)$. In our study, Chk1 was present both in the cytoplasm and the nucleus under normal conditions (Fig. 3B). Upon treatment with the Chk1-binding peptide, however, nuclear Chk1 was significantly decreased even in the absence of genotoxic treatment (Fig. 3C). Since Chk1 phosphorylation is slightly increased by treatment with the Chk1-binding peptide (Fig. 5B) under normal and IR conditions when compared to the controls, we hypothesized that the increased Chk1 phosphorylation was partly responsible for the decreased nuclear localization of Chk1. More importantly, considering the fact that the nuclear pool of Chk1 supports cell viability (22), the decreased cell viability of the Chk1-NP peptide-treated cells upon DNA damage may have been caused by the decreased nuclear pool of Chk1 (Figs. 4C and 5A).

The phosphorylation and subsequent activation of Chk1 lead to conformational changes and degradation of the protein (9). Our findings demonstrated that the expression levels of Chk1 were decreased in the Chk1-NP peptide treated cells upon 
DNA damage (Fig. 5B). The slightly increased phosphorylation of Chk1 in the Chk1-NP peptide treated cells may have triggered Chk1 degradation. Therefore, the decreased levels of Chk1, as well as the decreased nuclear pool of Chk1 may have caused decreased cell viability following genotoxic treatments in the Chk1-NP peptide-treated cells.

The enhanced activation of Chk1 results in the resistance of cancer cells to chemo- or radiotherapy (31-34). Therefore, the artificial inhibition of Chk1 activity may reverse such therapy resistance. The combination of Chk1 inhibition with chemo- or radiotherapy results in synthetic lethality in cancer therapy. As many tumors with defective p53 function depend on Chk1-dependent $\mathrm{S}$ and G2/M checkpoints for survival, such combination treatment should be more effective against p53-defective cancer cells $(35,36)$. In our study, the combination of Chk1-NP and genotoxic agents significantly decreased the survival of both HeLa and NCI-H460 cells (Figs. 4C and 5A). The combined treatment with IR and the peptide was slightly more effective in killing HeLa cells when compared to that of NCI-H460 cells (Fig. 5A). HeLa cells are p53-defective, whereas NCI-H460 cells are p53-functional. p53-functional NCI-H460 cells treated with Chk1-NP may better deal with the DNA damage response by mobilizing p53-dependent cell cycle checkpoints which may contribute to enhanced cell survival.

Of note, our findings demonstrated that treatment with the Chk1-NP peptide alone significantly decreased the viability of HeLa cells, but not that of NCI-H460 cells (Fig. 4A and B). These results are reminiscent of the study by Wang et al (29). In that study, the authors demonstrated that the expression of the constitutively active mutant form of Chk1 in the absence of DNA damage inhibited HeLa cell proliferation. In our study, the Chk1-binding peptide also showed decreased cell survival with increased Chk1 activation in HeLa cells (Figs. 4B and 5B). These findings suggest that the artificial activation of Chk1 in the absence of DNA damage by a specific Chk1-binding peptide like Chk1-NP may be utilized to develop a novel method in cancer therapy.

In conclusion, as demonstrated in the present study, although the use of a peptide as a therapeutic agent still presents many challenges, such as toxicity and stability of the peptide, the approach using a specific Chk1-binding peptide seems promising in enhancing the mechanistic understanding of Chk1 activities. The single or combined use of the Chk1-binding Chk1-NP peptide with chemo- or radiotherapy may provide a novel rationale for development of specific Chk1-targeting agents.

\section{Acknowledgements}

This study was supported by the nuclear research and development program through the National Research Foundation of Korea (NRF) funded by the Ministry of Science, ICT and Future Planning of Korea (grant no. NRF-2012M2A2A7012377).

\section{References}

1. Sancar A, Lindsey-Boltz LA, Unsal-Kaçmaz K and Linn S Molecular mechanisms of mammalian DNA repair and the DNA damage checkpoints. Annu Rev Biochem 73: 39-85, 2004.

2. Ciccia A and Elledge SJ: The DNA damage response: Making it safe to play with knives. Mol Cell 40: 179-204, 2010.
3. Smith J, Tho LM, Xu N and Gillespie DA: The ATM-Chk2 and ATR-Chk1 pathways in DNA damage signaling and cancer. Adv Cancer Res 108: 73-112, 2010.

4. Sørensen CS, Syljuåsen RG, Falck J, Schroeder T, Rönnstrand L, Khanna KK, Zhou BB, Bartek J and Lukas J: Chk1 regulates the $\mathrm{S}$ phase checkpoint by coupling the physiological turnover and ionizing radiation-induced accelerated proteolysis of Cdc25A. Cancer Cell 3: 247-258, 2003.

5. Falck J, Mailand N, Syljuåsen RG, Bartek J and Lukas J: The ATM-Chk2-Cdc25A checkpoint pathway guards against radioresistant DNA synthesis. Nature 410: 842-847, 2001.

6. Patil M, Pabla N and Dong Z: Checkpoint kinase 1 in DNA damage response and cell cycle regulation. Cell Mol Life Sci 70: 4009-4021, 2013.

7. Tapia-Alveal C, Calonge TM and O'Connell MJ: Regulation of chk1. Cell Div 4: 8, 2009.

8. Zhang Y and Hunter T: Roles of Chk1 in cell biology and cancer therapy. Int J Cancer 134: 1013-1023, 2014.

9. Zhang YW, Otterness DM, Chiang GG, Xie W, Liu YC, Mercurio F and Abraham RT: Genotoxic stress targets human Chk1 for degradation by the ubiquitin-proteasome pathway. Mol Cell 19: 607-618, 2005.

10. Leung-Pineda V, Huh J and Piwnica-Worms H: DDB1 targets Chk1 to the Cul4 E3 ligase complex in normal cycling cells and in cells experiencing replication stress. Cancer Res 69: 2630-2637, 2009.

11. Zhang YW, Brognard J, Coughlin C, You Z, Dolled-Filhart M, Aslanian A, Manning G, Abraham RT and Hunter T: The F box protein Fbx6 regulates Chk1 stability and cellular sensitivity to replication stress. Mol Cell 35: 442-453, 2009.

12. Dent P, Tang Y, Yacoub A, Dai Y, Fisher PB and Grant S: CHK1 inhibitors in combination chemotherapy: Thinking beyond the cell cycle. Mol Interv 11: 133-140, 2011.

13. Merry C, Fu K, Wang J, Yeh IJ and Zhang Y: Targeting the checkpoint kinase Chk1 in cancer therapy. Cell Cycle 9: 279-283, 2010.

14. Suganuma M, Kawabe T, Hori H, Funabiki T and Okamoto T: Sensitization of cancer cells to DNA damage-induced cell death by specific cell cycle G2 checkpoint abrogation. Cancer Res 59: 5887-5891, 1999.

15. Kawabe T: G2 checkpoint abrogators as anticancer drugs. Mol Cancer Ther 3: 513-519, 2004.

16. Zhao H, Watkins JL and Piwnica-Worms H: Disruption of the checkpoint kinase 1/cell division cycle 25A pathway abrogates ionizing radiation-induced $\mathrm{S}$ and $\mathrm{G} 2$ checkpoints. Proc Natl Acad Sci USA 99: 14795-14800, 2002.

17. Kim KS, Heo JI, Choi KJ and Bae S: Enhancement of cellular radiation sensitivity through degradation of Chk1 by the XIAP-XAF1 complex. Cancer Biol Ther 15: 1622-1634, 2014.

18. Saladin A, Rey J, Thévenet P, Zacharias M, Moroy G and Tufféry P: PEP-SiteFinder: a tool for the blind identification of peptide binding sites on protein surfaces. Nucleic Acids Res 42: W221-W226, 2014

19. Chen P, Luo C, Deng Y, Ryan K, Register J, Margosiak S, Tempczyk-Russell A, Nguyen B, Myers P, Lundgren K, et al: The 1.7 A crystal structure of human cell cycle checkpoint kinase Chk1: Implications for Chk1 regulation. Cell 100: 681-692, 2000

20. Zhao B, Bower MJ, McDevitt PJ, Zhao H, Davis ST, Johanson KO, Green SM, Concha NO and Zhou BB: Structural basis for Chk1 inhibition by UCN-01. J Biol Chem 277: 46609-46615, 2002.

21. Fuchs SM and Raines RT: Pathway for polyarginine entry into mammalian cells. Biochemistry 43: 2438-2444, 2004.

22. Wang J, Han X, Feng X, Wang Z and Zhang Y: Coupling cellular localization and function of checkpoint kinase 1 (Chk1) in checkpoints and cell viability. J Biol Chem 287: 25501-25509, 2012.

23. Dunleavy EM, Roche D, Tagami H, Lacoste N, Ray-Gallet D, Nakamura Y, Daigo Y, Nakatani Y and Almouzni-Pettinotti G: HJURP is a cell-cycle-dependent maintenance and deposition factor of CENP-A at centromeres. Cell 137: 485-497, 2009.

24. Sidi S, Sanda T, Kennedy RD, Hagen AT, Jette CA, Hoffmans R, Pascual J, Imamura S, Kishi S, Amatruda JF, et al: Chk1 suppresses a caspase-2 apoptotic response to DNA damage that bypasses p53, Bcl-2, and caspase-3. Cell 133: 864-877, 2008.

25. Parsels LA, Qian Y, Tanska DM, Gross M, Zhao L, Hassan MC, Arumugarajah S, Parsels JD, Hylander-Gans L, Simeone DM, et al: Assessment of chk1 phosphorylation as a pharmacodynamic biomarker of chk1 inhibition. Clin Cancer Res 17: 3706-3715, 2011. 
26. Katsuragi Y and Sagata N: Regulation of Chk1 kinase by autoinhibition and ATR-mediated phosphorylation. Mol Biol Cell 15: 1680-1689, 2004.

27. Scorah J, Dong MQ, Yates JR III, Scott M, Gillespie D and McGowan CH: A conserved proliferating cell nuclear antigeninteracting protein sequence in Chk1 is required for checkpoint function. J Biol Chem 283: 17250-17259, 2008.

28. Smits VA, Reaper PM and Jackson SP: Rapid PIKK-dependent release of Chk1 from chromatin promotes the DNA-damage checkpoint response. Curr Biol 16: 150-159, 2006.

29. Wang J, Han X and Zhang Y: Autoregulatory mechanisms of phosphorylation of checkpoint kinase 1. Cancer Res 72: 3786-3794, 2012.

30. Shimada M, Niida H, Zineldeen DH, Tagami H, Tanaka M, Saito $\mathrm{H}$ and Nakanishi M: Chk1 is a histone $\mathrm{H} 3$ threonine 11 kinase that regulates DNA damage-induced transcriptional repression. Cell 132: 221-232, 2008.

31. Perego P, Gatti L, Righetti SC, Beretta GL, Carenini N, Corna E, Dal Bo L, Tinelli S, Colangelo D, Leone R, et al: Development of resistance to a trinuclear platinum complex in ovarian carcinoma cells. Int J Cancer 105: 617-624, 2003.
32. Bao S, Wu Q, McLendon RE, Hao Y, Shi Q, Hjelmeland AB, Dewhirst MW, Bigner DD and Rich JN: Glioma stem cells promote radioresistance by preferential activation of the DNA damage response. Nature 444: 756-760, 2006.

33. Bartucci M, Svensson S, Romania P, Dattilo R, Patrizii M, Signore M, Navarra S, Lotti F, Biffoni M, Pilozzi E, et al: Therapeutic targeting of Chk1 in NSCLC stem cells during chemotherapy. Cell Death Differ 19: 768-778, 2012.

34. Wang X, Ma Z, Xiao Z, Liu H, Dou Z, Feng X and Shi H: Chk1 knockdown confers radiosensitization in prostate cancer stem cells. Oncol Rep 28: 2247-2254, 2012.

35. Koniaras K, Cuddihy AR, Christopoulos H, Hogg A and O'Connell MJ: Inhibition of Chk1-dependent G2 DNA damage checkpoint radiosensitizes p53 mutant human cells. Oncogene 20: 7453-7463, 2001

36. Ma CX, Cai S, Li S, Ryan CE, Guo Z, Schaiff WT, Lin L, Hoog J, Goiffon RJ, Prat A, et al: Targeting Chk1 in p53-deficient triple-negative breast cancer is therapeutically beneficial in human-in-mouse tumor models. J Clin Invest 122: 1541-1552, 2012. 